\title{
Genetic Algorithm Optimization of the Volute Shape of a Centrifugal Compressor
}

\author{
Martin Heinrich and Rüdiger Schwarze \\ Institute of Mechanics and Fluid Dynamics, Technical University Bergakademie Freiberg, \\ 09599 Freiberg, Germany \\ Correspondence should be addressed to Martin Heinrich; martin.heinrich@imfd.tu-freiberg.de
}

Received 30 December 2015; Accepted 21 March 2016

Academic Editor: Farid Bakir

Copyright (c) 2016 M. Heinrich and R. Schwarze. This is an open access article distributed under the Creative Commons Attribution License, which permits unrestricted use, distribution, and reproduction in any medium, provided the original work is properly cited.

A numerical model for the genetic optimization of the volute of a centrifugal compressor for light commercial vehicles is presented. The volute cross-sectional shape is represented by cubic B-splines and its control points are used as design variables. The goal of the global optimization is to maximize the average compressor isentropic efficiency and total pressure ratio at design speed and four operating points. The numerical model consists of a density-based solver in combination with the SST $k-\omega$ turbulence model with rotation/curvature correction and the multiple reference frame approach. The initial validation shows a good agreement between the numerical model and test bench measurements. As a result of the optimization, the average total pressure rise and efficiency are increased by over $1.0 \%$ compared to the initial designs of the optimization, while the maximum efficiency rise is nearly $2.5 \%$ at $\dot{m}_{\text {corr }}=0.19 \mathrm{~kg} / \mathrm{s}$.

\section{Introduction}

Due to increasing environmental awareness and stricter pollution policies, the demand for low carbon vehicles is steadily growing. This results in highly efficient and downsized engines. One key element for this development is the turbocharger which helps to reduce the engine size, increase efficiency, and lower the emissions.

A typical compressor of a turbocharger for passenger cars and light commercial vehicles consists of an inlet port, the compressor wheel, and the volute with an exit pipe. The compressor wheel is well understood and investigated intensively throughout the recent decades [1-4]. On the contrary, the volute is less investigated. Still, this part plays an important role in the compressor flow due to the following reasons [5]: (1) the influence of the overall performance of the centrifugal compressor, (2) the pressure distortion at off-design conditions, and (3) effect on the stability limits of the compressor. Furthermore, Worster [6] states that the volute determines the point of best efficiency of pumps and compressors rather than the compressor wheel. Therefore, it is vital to understand the influence of design parameters onto the volute and compressor performance.

Investigations of different volute geometric parameters were performed as early as the 1940s. However, experimental investigations of different volute geometry configurations are limited due to production costs and time. In recent years, more research was conducted regarding the volute design in compressors, fans, and pumps using numerical simulations [7-11]. One advantage is that designs can be tested virtually and no production costs arise. Nonetheless, the total number of investigations in open literature concerning the volute geometry is small.

Literature Review. Due to the increase in computational power, mathematical optimization is more and more utilized in the design process for turbomachinery application. Figure 1 shows the number of publications per year listed in Scopus [12] dealing with optimization of compressors using CFD. Since the beginning of the last decade, the number of research works on this topic is steadily increasing. This is the result of the rising demand for higher performance 


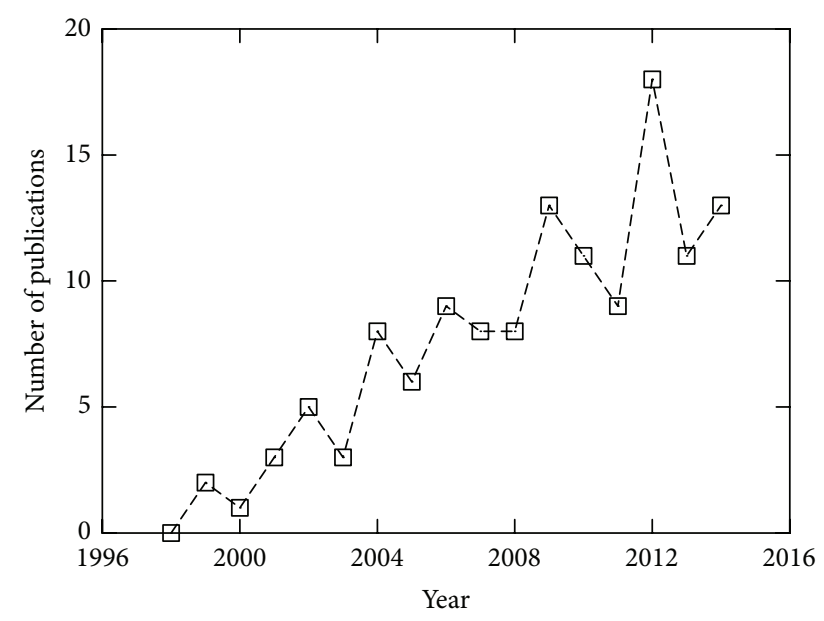

FIGURE 1: Number of publications regarding the numerical optimization of compressors using CFD per year in Scopus [12].

turbomachines with better efficiencies and lower environmental impact. Due to the complexity of modern propulsion systems, these improvements cannot be achieved through case studies any more. For this reason, optimization based on mathematical models and numerical simulations has to be employed. Although the given overview consists of only Scopus cited publications, the trend is, nevertheless, noticeable and the total number of research works on this topic in both academia and industry is going to be higher.

Despite an increase in CFD-based optimization, the vast amount of research work is unilaterally focused on the improvement of the compressor wheel and blade design. Over $86 \%$ of the investigated publications are related to this part of the compressor. One reason is the simpler geometry and mesh generation for impeller. Blade geometries can be created easily using specialized commercial tools like ANSYS $^{\circledR}$ BladeGen and ANSYS TurboGrid. Furthermore, parametrization of the cross-sectional shape can be achieved using splines in combination with extrusion in pitchwise direction. This allows for fast geometry and mesh generation, which is essential for automatic optimization. Additionally, simplifications such as two-dimensional or rotationally symmetrical simulations can be used to quickly assess blade performance in axial and radial machines.

By contrast, only a small fraction of optimization work was performed on the volute geometry. The automated volute geometry generation and meshing are very difficult due to the complex shape. Furthermore, no geometrical simplifications can be made and the complete three-dimensional volute has to be simulated. This often includes the compressor wheel upstream the volute to incorporate interactions between both parts. Therefore, it is more time consuming compared to blade or impeller investigations. For these reasons, research work on the field of volute optimization is very limited or restricted to simplified geometric variations. Important work regarding this topic is presented in the following.

Jang et al. [13] numerically analysed a centrifugal blower with a flow coefficient of 0.139 , a pressure coefficient of 1.29 ,
TABLE 1: Experimental results of reference and optimized volute of Baloni et al. [14].

\begin{tabular}{lcc}
\hline Performance parameter & Reference volute & $\begin{array}{c}\text { Optimized } \\
\text { volute }\end{array}$ \\
\hline Flow coefficient & 0.245 & 0.266 \\
Pressure coefficient & 0.69 & 0.74 \\
Efficiency & $46.7 \%$ & $54.1 \%$ \\
\hline
\end{tabular}

and an efficiency of $78.3 \%$ at design point. The impeller has an exit diameter of $1625 \mathrm{~mm}$ at a design speed of $1780 \mathrm{~min}^{-1}$. The volute has a trapezoidal shape with a symmetrical inlet (see Figure 2). The height and width are used as design parameters with reference values of $300 \mathrm{~mm}$ and $560 \mathrm{~mm}$, respectively. The pressure rise and isentropic efficiency are defined as objective functions. The experimental points are determined using design of experiments (DoE) and the objective function in each point is evaluated using a steadystate simulation. Finally, the response surface is calculated using a second-order polynomial.

The blower pressure coefficient and efficiency of the optimized model are increased by $1.6 \%$ and $0.4 \%$, respectively, compared to the reference model. The total variation for different width and heights is approximately $6 \%$ for the efficiency. The main reason for the difference in efficiency is identified to be secondary flow in the corners.

Baloni et al. [14] investigated the volute design using the Taguchi method of optimization and Analysis of Variance (ANOVA) approach. The work is based on the numerical simulation of a centrifugal blower with an exit diameter of $425 \mathrm{~mm}$ at a design speed of $2900 \mathrm{~min}^{-1}$. The flow coefficient and pressure coefficient of the reference model are 0.245 and 0.69 , respectively. Three geometric parameters are defined as design variables: blower volute width, tongue angle, and radial position of the volute. The objective function is composed of the variation of static pressure at impeller outlet, losses inside the volute, and total pressure at volute outlet. The simulations are performed using a steady-state approach. The numerically optimized geometry is validated using test bench measurements.

The improvements of the optimized volute geometry are shown in Table 1 . The increase in efficiency is significant with about 7.4\%. However, the baseline efficiency is quite low with $46.7 \%$. Therefore, improvements are more feasible. The researchers suggest a blower width of 1.5 times the impeller width for an optimum performance. Furthermore, the tongue angle should be $26^{\circ}$ for minimizing static pressure variations at the impeller outlet and $24^{\circ}$ for reducing the total pressure at the volute outlet.

Huang et al. [15] optimized a volute with rectangular cross-sectional shape for a centrifugal compressor. The volute shape consists of five different geometric parameters $L_{1, \ldots, 5}$ (see Figure 3(a)). These were defined at an azimuthal angle of $45^{\circ}$ and $360^{\circ}$. Linear interpolation is employed for the shapes in between. In total, seven different design parameters are chosen. A constrained optimization using response surfaces is used for the improvement of the geometry. The objective 


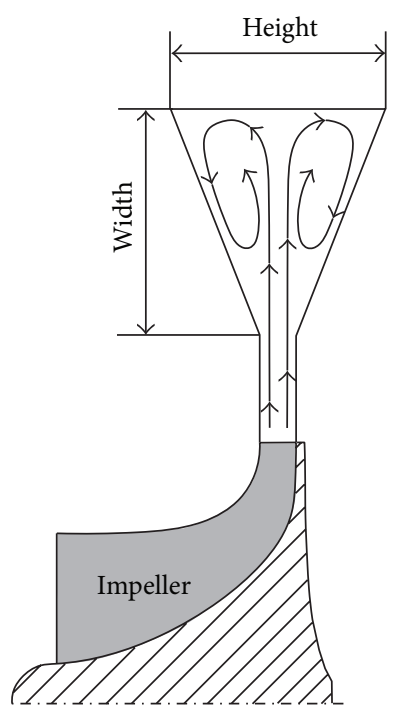

(a)

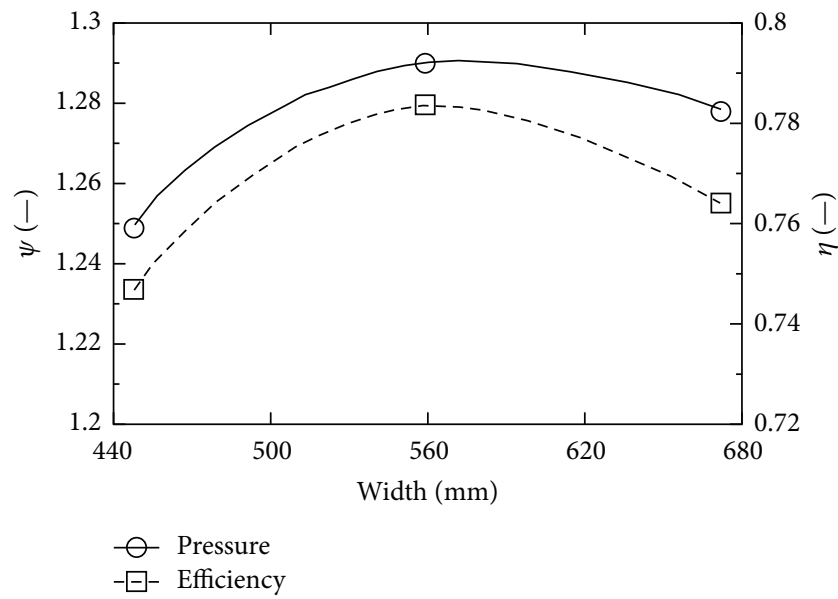

(b)

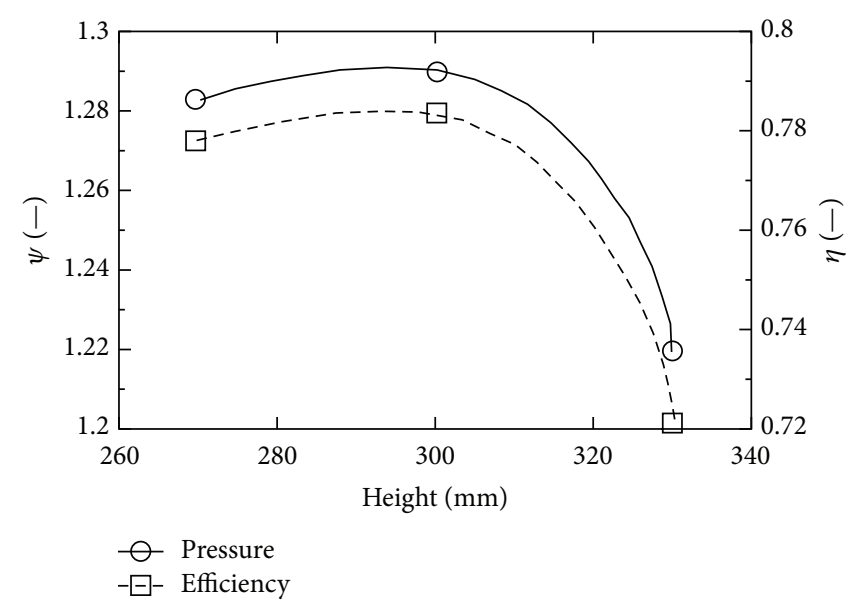

(c)

FIGURE 2: Volute cross-sectional shape and geometric parameters (a) and blower performance for varying width at reference height of $300 \mathrm{~mm}$ (b) and varying height at reference width of $560 \mathrm{~mm}$ (c) according to Jang et al. [13].

function is to maximize the minimal value of the volute static pressure coefficient at three different flow rates: $90 \%, 100 \%$, and $110 \%$ of design flow rate.

The simulations are performed using a simplified voluteonly model. The averaged volute inlet boundary conditions are taken from full-stage simulations of the complete compressor. Matlab is used for the optimization process. Thirty-five initial variations are used for the start. After 120 iterations, the optimization process converges. As a result, the efficiency of the volute is increased by about $6 \%$ (see Figure 3(b)) and the total pressure loss coefficient is reduced by about 0.04 . However, this leads just to a minor increase of polytropic stage efficiency of $0.4 \%$. The reason for this improvement is reported to be the corner cut-off at $L_{3}$ and $L_{4}$, which decreases losses due to secondary vortices.

Despite the significance of the volute geometry, research work regarding the numerical optimization of this part of a centrifugal compressor is very limited. Furthermore, the proposed numerical models are simplified or do not offer large geometric variety. This paper tries to fill this gap by presenting the genetic optimization of the cross-sectional shape of the volute of a centrifugal compressor. The surface is represented by B-splines offering a tremendous amount of different shapes necessary for optimization. The goal is the improvement of the compressor total pressure ratio and isentropic efficiency.

\section{Volute Geometry Generation}

One essential requisite for a numerical optimization is the automated geometry and mesh generation. The former is especially challenging in case of a volute. In contrast to blades, the creation of a volute geometry is much more complex due to the nonsymmetrical, fully three-dimensional shape and the complex tongue. For these reasons, a design 


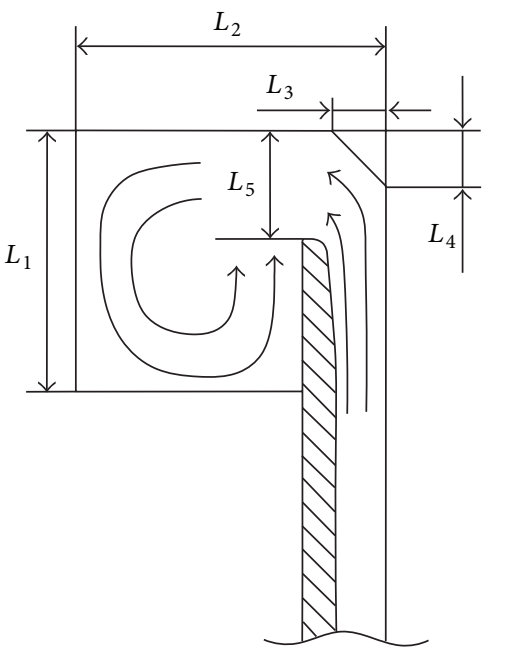

(a)

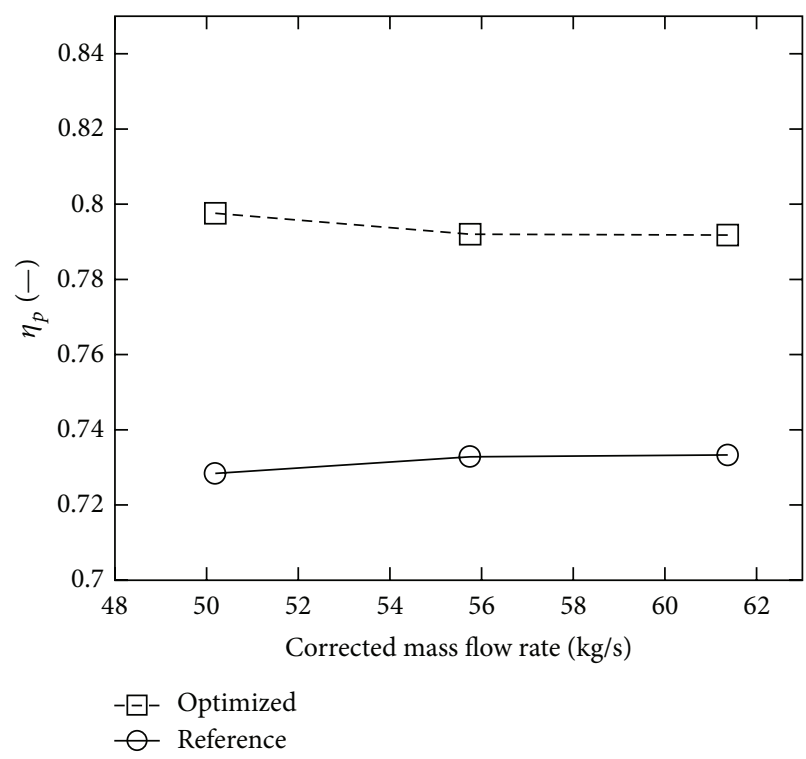

(b)

FIGURE 3: Five geometric parameters of volute shape (a) and polytropic efficiency $\eta_{p}$ of the reference and optimized volute geometry (b) as a function of the mass flow rate $\dot{m}_{\text {corr }}$ according to Huang et al. [15].

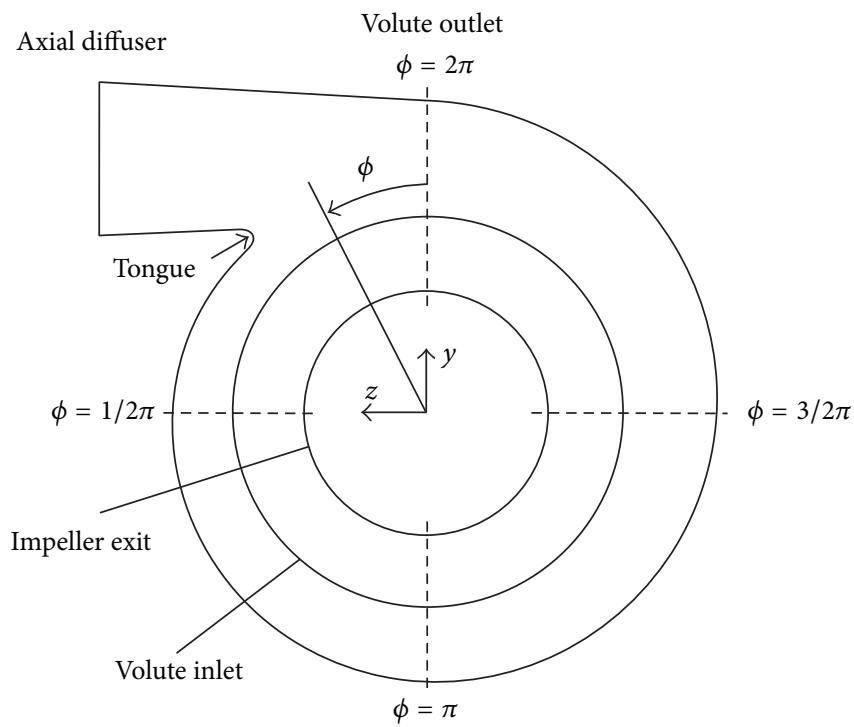

(a)

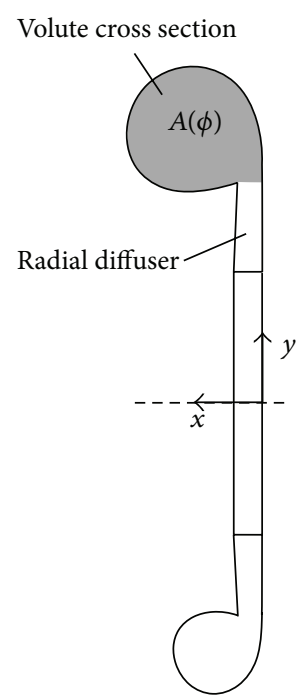

(b)

FIGURE 4: Definition of the volute geometry in top-down view (a) and sectional view through the point of origin (b).

program for the automated generation of the volute geometry is developed. It is written in the object-oriented programming language $\mathrm{C}++$. The tool can be integrated easily with other simulation software and the creation of the geometry is completely automated and does not require any additional user intervention. Therefore, it is ideal for automated studies and optimization. The geometric definition of the volute can be seen in Figure 4.
Due to the elaborate shape of the volute, simple polynomial functions do not suffice for the geometry construction. Likewise, the combination of basic geometric shapes does not give the variety needed for an optimization. For this reason, B-splines are employed to represent and most importantly parameterize the volute surface. These curves offer a large range of geometric freedom while using only a few parameters. 


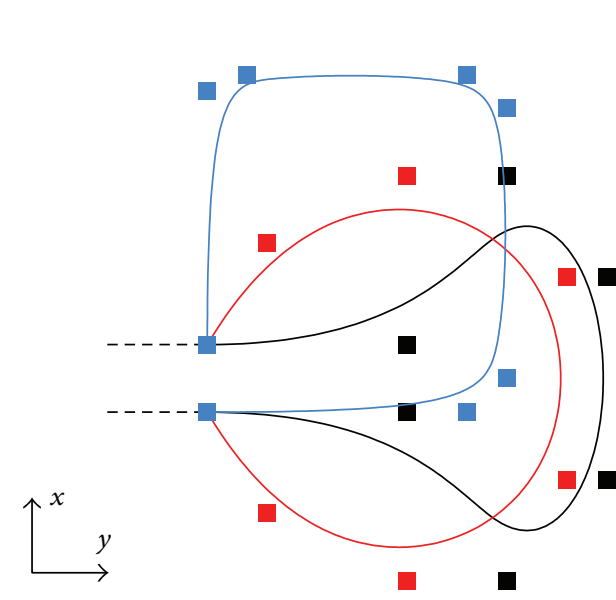

(a) Shape variation

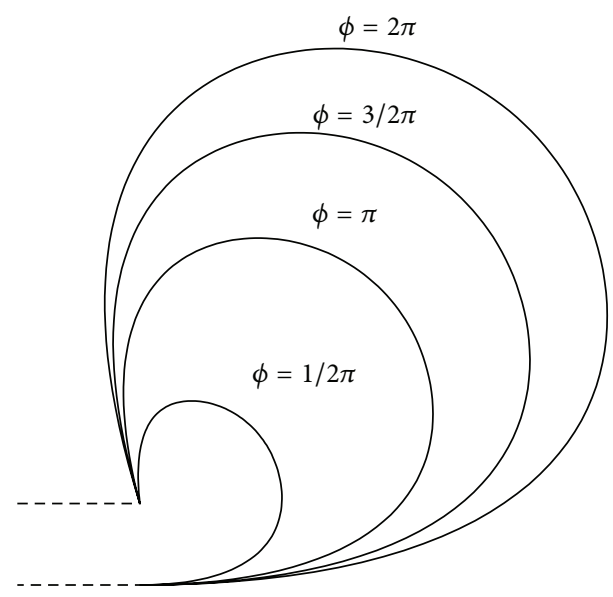

(b) Scaling

FIGURE 5: Three different volute shapes represented by B-splines using eight control points (a) and scaling of the volute shape cross-sectional areas as a function of the azimuthal angle $\phi(b)$.

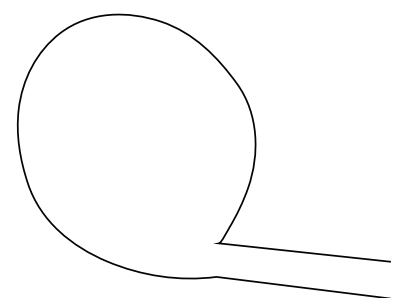

(a) Initial spline generation

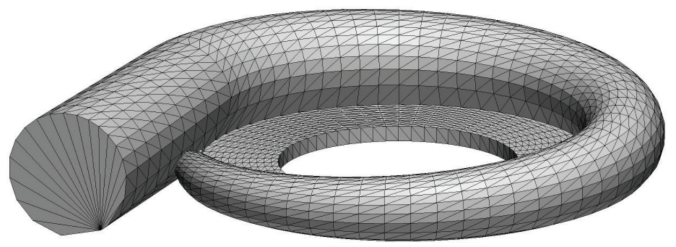

(c) Surface mesh with sharp tongue

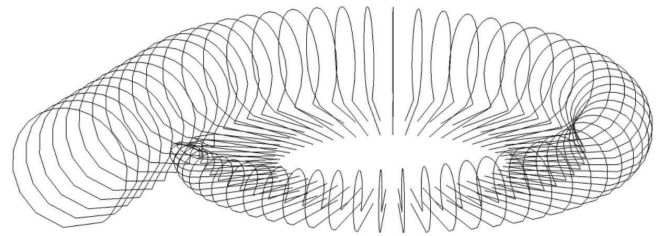

(b) Duplicated and rotated splines

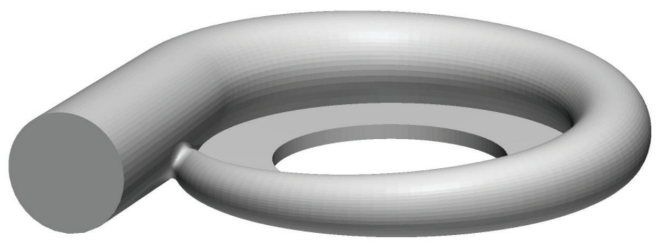

(d) Smooth surface with rounded tongue

FIGURE 6: Steps of volute geometry. The surface mesh and spline representation are simplified for better illustration.

The cross-sectional shape of the volute is represented by a third-order B-spline with eight control points (see Figure 5(a) for three examples). By altering the coordinates of these points, the shape of the spline changes. Therefore, a wide range of different geometries can be assembled. More complex shapes could be achieved by adding control points to the spline. However, their number is a trade-off between the geometric freedom of possible shapes and the number of adjustable parameters for the optimization. The more the parameters are, the more complex and computationally expensive the optimization will be.

The cross-sectional area of the volute channel is a linear function of the azimuth angle. In order to match a specific cross-sectional area, the volute shape is scaled based on the azimuth angle $\phi$ as shown in Figure 5(b). In order to change the size, the control points are shifted towards the volute inlet. In this way, the basic geometrical shape is preserved while adjusting the size of the volute channel.
The automated generation of the volute can be summarized in four steps, which are shown in Figure 6. (a) At first, based on the user input, the volute cross section and the radial diffuser are created at $\phi=2 \pi$. (b) The volute splines are duplicated and rotated in circumferential direction with respect to the rotation axis of the compressor wheel and their size is adjusted to match the volute cross-sectional area. Additionally, the axial diffuser is extruded from the volute exit shape and morphed along the axial direction to form a circular diffuser exit. (c) Based on this, triangulated surfaces in STL format (stereolithography) are created for each single part of the volute. (d) The fillet surface is generated by calculating the intersection between volute and axial diffuser surface and forming a smooth fillet at this location.

The open-source meshing tool snappyHexMesh, which is part of the open-source CFD-library OpenFOAM version 2.4.0 [16], is used for the volume mesh generation of the volute (see Figure 7). This program automatically creates an 


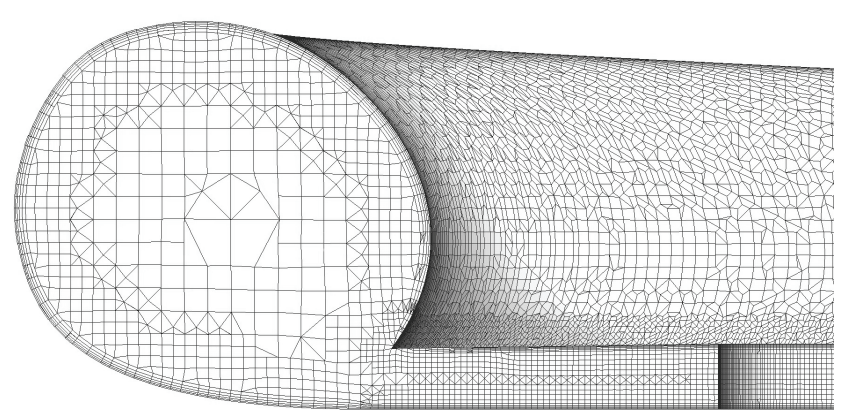

FIGURE 7: Slice of the unstructured hexahedral-dominant mesh with prism layers automatically generated using snappyHexMesh at the volute exit.

unstructured hexahedral mesh based on the STL surfaces as input geometries. For this reason, it integrates very well with the automated geometry generation. Five prism cells are used to model the boundary layer at the wall. This results in an average dimensional wall distance of $y^{+}=16-24$.

\section{Numerical Model of the Centrifugal Compressor}

3.1. Geometry and Mesh. The turbocharger was designed for light commercial vehicles. Its design point is at a rotational speed of $n=100,000 \mathrm{~min}^{-1}$, which is equivalent to a circumferential velocity of $340 \mathrm{~m} / \mathrm{s}$ at the trailing edge at $65 \mathrm{~mm}$ diameter. This corresponds to a corrected mass flow rate of $\dot{m}_{\text {corr }}=0.120 \mathrm{~kg} / \mathrm{s}$ of air, a total pressure ratio of $\Pi_{t t}=2.05$, and an isentropic efficiency of the compressor side of $\eta_{\text {is }}=0.736$. The impeller consists of 7 blade sets, each containing one main blade and one splitter blade at a constant tip clearance of $0.3 \mathrm{~mm}$. The vaneless diffuser has a tapering span with a minimum height of $4 \mathrm{~mm}$.

Some geometry simplifications are made to reduce the complexity of the meshing process and obtain accurate results in a reasonable time frame. Firstly, the fillet radius between the blades and the hub of the compressor wheel is neglected. This increases the modelled flow cross-sectional area in the impeller by about $2 \%$. Secondly, the gap between the compressor wheel and the diffuser is ignored. For that reason, the influence of the backside cavity of the impeller cannot be accounted for.

The computational domain is divided into three different regions (see Figure 8). Due to the complex geometry, the inlet domain is meshed using a tetrahedral/prism hybrid mesh with 5 prism layers at the walls. The impeller is meshed using a block structured hexahedral mesh. The inlet domain and the compressor wheel are connected to the volute mesh from the previous chapter using arbitrary mesh interfaces. These allow the use of nonconformal meshes at the interfaces and therefore reduce the meshing expense. The mesh is locally refined at crucial regions, such as leading edge of the impeller blades, the tip clearance, and the tongue of the volute.

Four different meshes were used in order to investigate the mesh dependency of the numerical model (see Table 2).
Inlet domain:

(i) Tetra/prism hybrid mesh

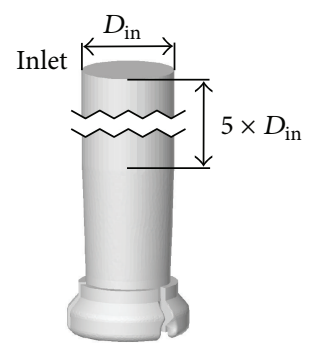

Impeller (MRF-zone):

(i) Structured hexahedral mesh

Volute domain:

(i) Unstructured hexahedral mesh

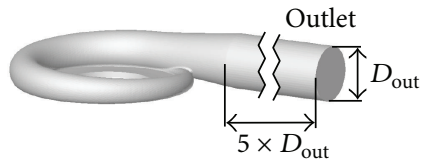

FIgURE 8: Computational domain of the compressor.

TABle 2: Cell count for the grid dependency study and average nondimensional wall distance $\bar{y}^{+}$for the near-wall cells.

\begin{tabular}{lccccc}
\hline $\begin{array}{l}\text { Mesh } \\
\text { region }\end{array}$ & Coarse & Normal & Fine & $\begin{array}{l}\text { Very } \\
\text { fine }\end{array}$ & $\bar{y}^{+}$ \\
\hline Inlet & 0.49 & 0.72 & 0.96 & 1.50 & 24 \\
Impeller & 1.00 & 1.50 & 2.00 & 3.00 & 16 \\
Volute & 0.52 & 0.76 & 0.99 & 1.48 & 24 \\
\hline$\sum$ & 2.01 & 2.98 & 3.95 & 5.98 & \\
\hline
\end{tabular}

The mesh size is $(2.01-5.98) \times 10^{6}$ cells. About $50 \%$ of all cells are located inside the compressor wheel part due to the complexity of the flow. The average dimensionless near-wall distance $\bar{y}^{+}$is nearly constant for each mesh with 24 for the inlet and volute region and 16 for the impeller.

The turbocharger compressor inlet and outlet have diameters of $65 \mathrm{~mm}$ and $42 \mathrm{~mm}$, respectively. The inlet pipe upstream the ported shroud and the outlet pipe downstream the volute are extended using an extrusion mesh, in order to reduce the influence of the inflow and outflow boundary conditions onto the main flow. Furthermore, this also matches the inlet and outlet boundaries with the measurement positions of the test bench.

3.2. Numerical Model. A density-based solver is used for the simulation of the radial compressor (see Heinrich and Schwarze [17] for more details). The Simple Low-dissipation AUSM (SLAU) scheme introduced by Kitamura and Shima $[18,19]$ is employed for the flux calculation with Weiss-Smith preconditioning and local time stepping. Spatial discretization is second order. The SST $k-\omega$ model with rotation and curvature correction [20] models the influence of turbulence onto the flow in combination with wall functions. The air is defined as perfect gas with Sutherland's law for viscosity modelling. The multiple reference frame (MRF) approach is used to account for the rotation of the compressor wheel.

The flow is considered to be steady state. Transient simulations are run until a steady-state solution is achieved. 
TABLE 3: Inlet and outlet boundary conditions.

\begin{tabular}{lcc}
\hline \multirow{4}{*}{ Inlet } & $p_{\text {tot }}$ & $101325 \mathrm{~Pa}$ \\
& $T_{\text {tot }}$ & $297.15 \mathrm{~K}$ \\
& $I_{t}$ & $10 \%$ \\
Outlet & $L_{t}$ & $5 \mathrm{~mm}$ \\
& $p$ & Varying \\
& Others & Zero gradient \\
\hline
\end{tabular}

Thereby, the simulations can give an accurate performance prediction for the design point and the choke limit. This way a major part of the performance map can be investigated. Oscillations in the flow patterns indicate an unstable operating point. This is particularly the case for low mass flow rates in combination with high pressure ratios, where rotating stall leads to temporal fluctuations of the mass flow. These fluctuations cannot be physically resolved with the steady-state approach. The solution convergence is evaluated using several monitor points throughout the domain. Furthermore, the static pressure and temperature at the outlet are monitored. Convergence is achieved, when the monitored quantities reach a constant value. In case of unsteady behaviour or oscillations, the flow field is averaged over the iterations and the mean field is used for postprocessing.

The boundary conditions are chosen to match the parameters of the test bench measurements. The stagnation pressure at the inlet is set to $101,325 \mathrm{~Pa}$ with a temperature of $297.15 \mathrm{~K}$. At the outlet, the mass flow according to the experiments is specified. Other variables at the outlet are treated with zero gradient. The walls are defined as adiabatic and surface roughness is not considered. The boundary conditions are summarized in Table 3.

3.3. Genetic Optimization Procedure. A single objective genetic optimization is performed using the software toolkit Dakota [21]. The chosen optimization method is from the SCOLIB library called coliny_ea. The objective function $f$ is the arithmetic average of the isentropic efficiency $\eta_{\text {is }}$ at design speed $n=100,000 \mathrm{~min}^{-1}$ for four operating points at $(0.12-0.19) \mathrm{kg} / \mathrm{s}$ mass flow rate:

$$
f=\frac{1}{4} \cdot \sum_{i=1}^{4} \eta_{\mathrm{is}, i} .
$$

This way, the performance of the compressor is maximized for a specific operating range, not at design point only. The design parameters of the optimization are the coordinates of the eight control points of the two-dimensional volute cross-sectional spline. Since the first and last control point are fixed, this results in 12 modifiable parameters. Upper and lower bounds are used to limit the points and ensure feasible designs. Only the volute shape is changed, while the radial and axial diffuser as well as the tongue of the volute remain unchanged. Therefore, improvements of the compressor performance can be related to changes in volute shape. Each set of design parameters results in one unique volute geometry. The generations during the optimization consist of 50 individuals. The initial generation is made of

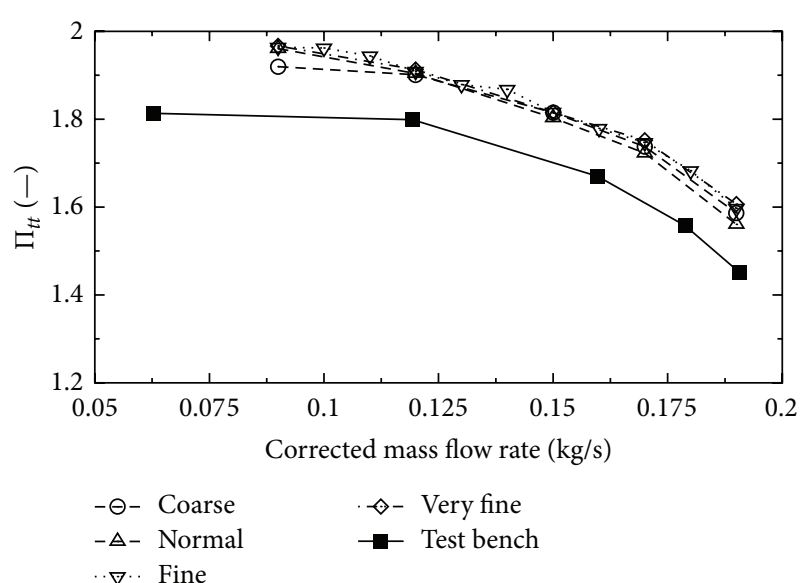

(a)

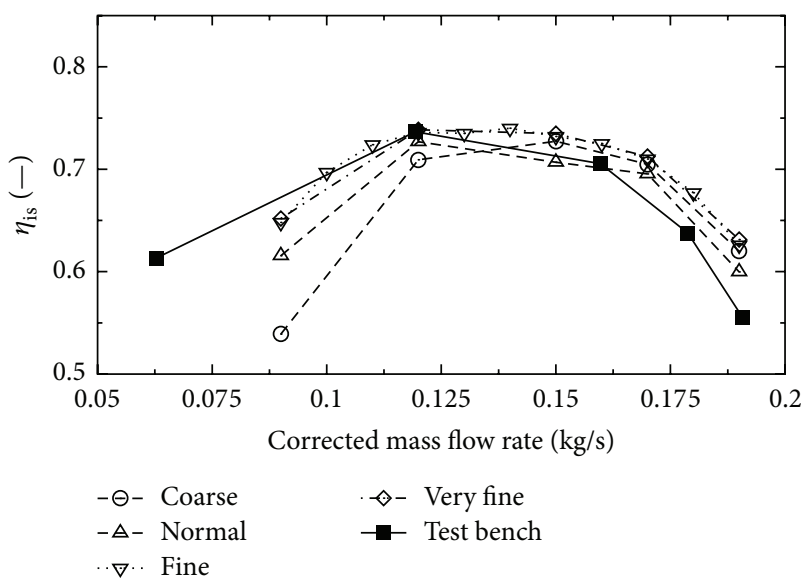

(b)

FIGURE 9: Total pressure ratio and isentropic efficiency for different grid resolutions.

predefined and randomly generated volute shapes to start with a large diversity. The mutation rate is $30 \%$ and the crossover rate $80 \%$. This high mutation rate reduces the chance that the optimization algorithm does get stuck in local extrema.

\section{Results}

4.1. Grid Dependency. The influence of the different grid resolutions is shown in Figure 9. The total pressure ratio as well as the isentropic efficiency is plotted for a cell count of (2.01-5.98) $\times 10^{6}$. The simulation represents the measured total pressure ratio well and the slopes are met very well. The reference values are overestimated by approximately $5 \%$. The influence of the cell count is minor. However, the coarse and normal mesh show slightly lower pressure ratios at high and low flow rates compared to the fine and very fine mesh. Similar results can be seen at the isentropic efficiency. The coarse and normal meshes show a significant drop in efficiency and strongly underpredict the test bench measurements. One can suspect that the coarse grids cannot resolve the complex flow 


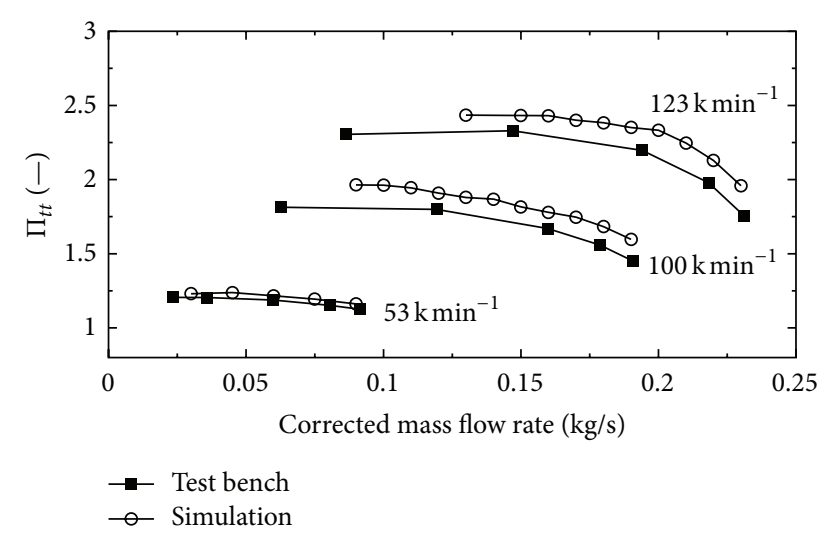

(a)

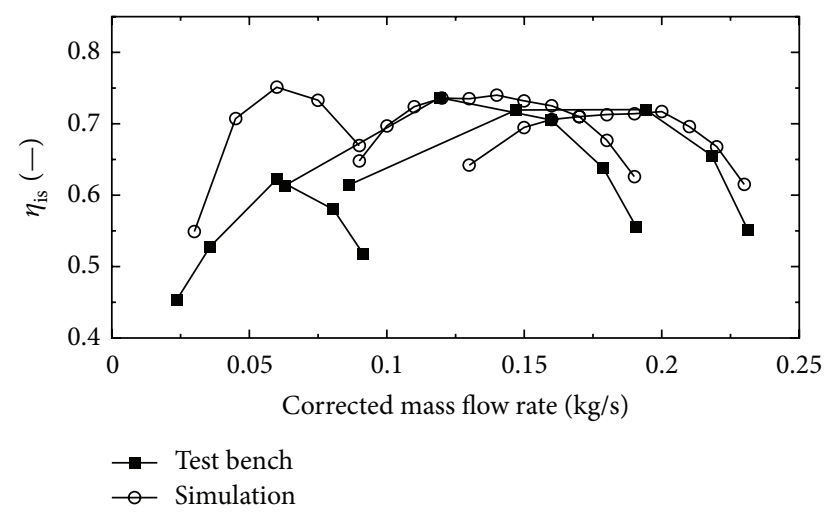

(b)

FIGURE 10: Total pressure ratio $\Pi_{t t}$ and isentropic efficiency $\eta_{\text {is }}$ for the simulation and test bench measurements.

inside the compressor sufficiently leading to additional losses. Again, the fine and very fine mesh offer similar results. This indicates that a mesh resolution of $4 \times 10^{6}$ cells is sufficient for an almost mesh independent solution. Therefore, the fine grid is used for further investigations of the compressor. It offers the best compromise between accuracy and computational cost.

4.2. Investigation of Baseline Geometry. The total pressure ratio for the innoSTREAM turbocharger for the three different rotational speeds is presented in Figure 10(a). In general, all numerical results show a good accordance to the test bench measurements. The total pressure ratio is overpredicted for all operating conditions. The difference is small at lower angular velocities with less than $3 \%$ at $53,000 \mathrm{~min}^{-1}$. However, the total pressure ratio at the higher speeds is overestimated by up to $6 \%$. Nevertheless, the trends of the performance map are computed very well and the choke limit is predicted correctly. Figure 10(b) shows the isentropic efficiency as a function of the mass flow rate. It is computed very well for $100,000 \mathrm{~min}^{-1}$ and $123,000 \mathrm{~min}^{-1}$. However, at low speeds, the isentropic efficiency is significantly overpredicted.

Sources for the differences between experiment and simulation are diverse such as geometry and modelling

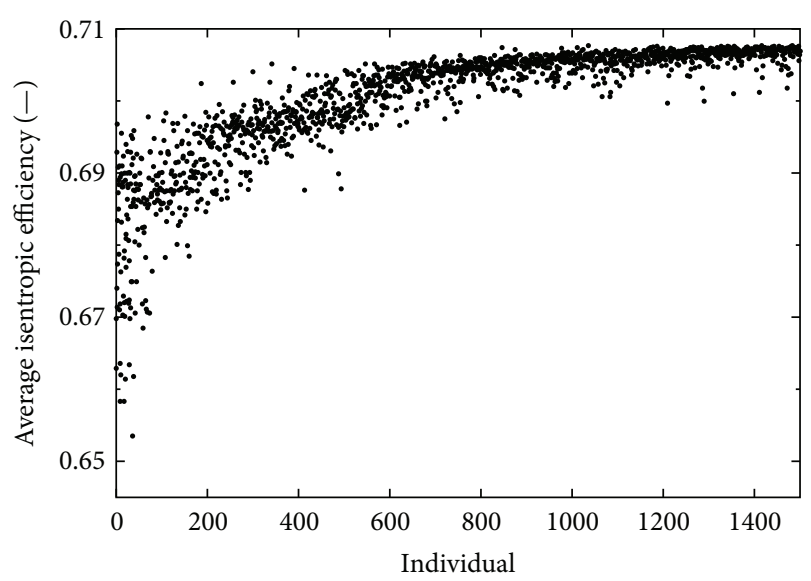

FIGURE 11: Average isentropic efficiency for each individual.

simplifications. The fillet radius between the blade and the hub is neglected. This increases the effective cross-sectional area and thus allows for higher flow rates. According to [22], the flow in the impeller backside cavity causes additional losses in efficiency and total pressure ratio of about $1 \%$. This effect is also not captured in the simulations. Another source of error is the lack of real mesh movement. Therefore, blade passage effects are not resolved.

The isentropic efficiency for low speeds is significantly overestimated. According to Bohn et al. [23], this can be attributed to the heat transfer from the turbine side of the turbocharger. This heat transfer is mainly composed of heat radiation from the hot surface and heat conduction via the housing and the shaft that connects the turbine and the compressor wheel (see Verstraete et al. [24]). This additional heat input increases the compressor air outlet temperature and reduces the efficiency. The main reason for these differences is the definition of the walls as adiabatic. This suppresses the heat transfer to the surroundings, implying a higher ambient temperature. Therefore, the computed outlet gas is hotter than the experimental data leading to a lower isentropic efficiency.

Operating points close to the surge cannot be resolved using the presented model. Hellstrom et al. [2] characterized the flow near stall as dominated by highly unsteady flow separation at the rotor as well as reversed in the inlet region and ported shroud. These effects cannot be resolved using a steady-state simulation. Furthermore, Baris and Mendonca [25] argue that wall-function RANS-based simulation fails to predict flow separation, which is characteristic for low mass flow regimes.

4.3. Genetic Optimization. During the genetic optimization, 30 generations were created with 1500 individual volute shapes. The average isentropic efficiency for each individual is shown in Figure 11. The biggest increase can be seen in the first few hundred individuals. After approximately 20 generations, the stage performance of the compressor stays nearly constant. During the optimization, the shapes for each generation vary strongly. During the first generations 

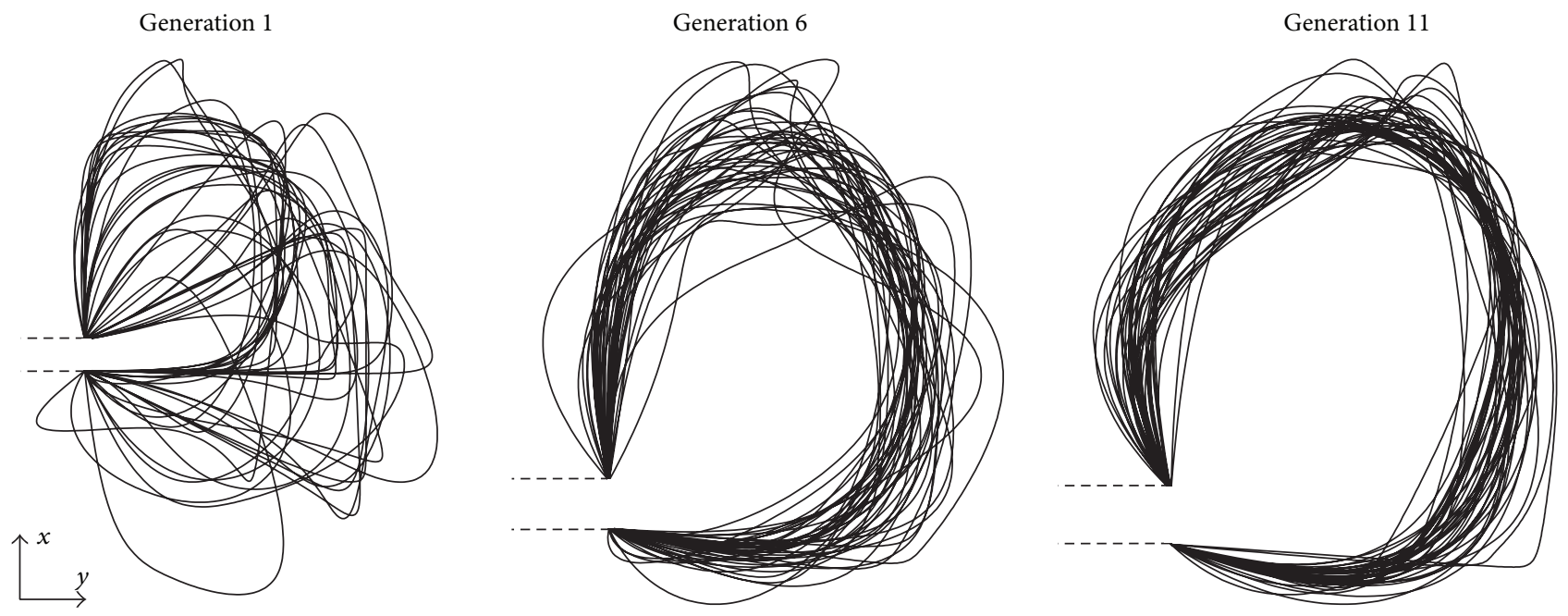

FIGURE 12: Cross-sectional volute shapes for different generations during the optimization.

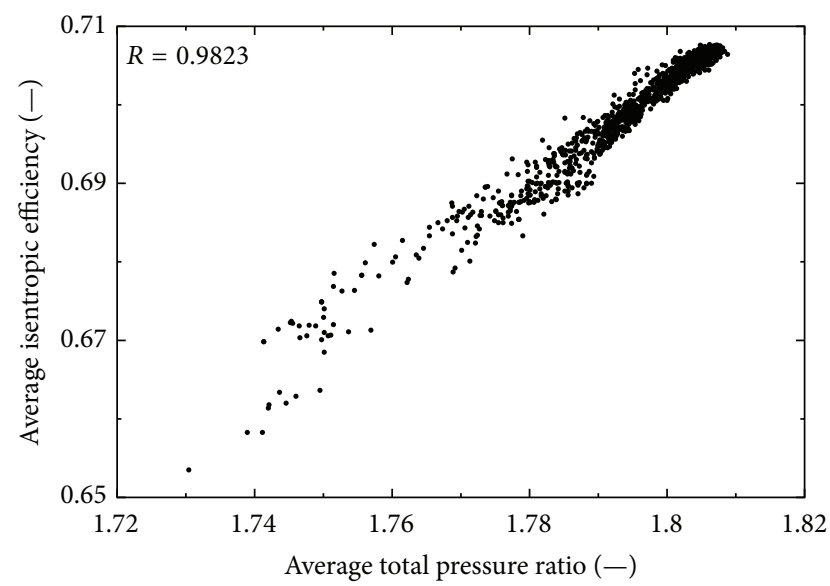

FIGURE 13: Average isentropic efficiency against the average total pressure ratio for different individuals. The correlation coefficient $r$ is nearly one which indicates a linear correlation.

(see Figure 12), there is a significant diversity of forms due to many different shapes in the first generation. However, as the genetic optimization progresses, poor performing individuals are sorted out and fitter shapes start to dominate. After 11 generations, a trend towards circular-shaped cross sections is noticeable.

Using only the average isentropic efficiency as function object for the optimization is only valid if both the total pressure ratio and the efficiency are linearly correlated. Otherwise a multiobjective optimization would be preferred for finding the best volute geometry. Figure 13 shows both quantities for different cross-sectional shapes. The results reveal a linear correlation between total pressure ratio and isentropic efficiency. For this reason, it is sufficient to maximize one of the two parameters. This is also supported by Baloni and Channiwala [26] stating that the volute does not take part in the energy transfer onto the fluid and all considerations

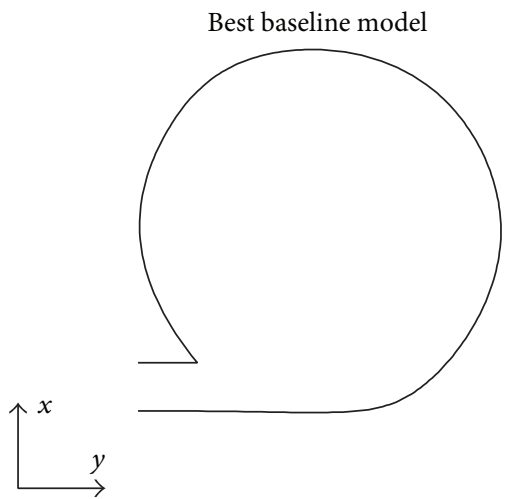

(a)

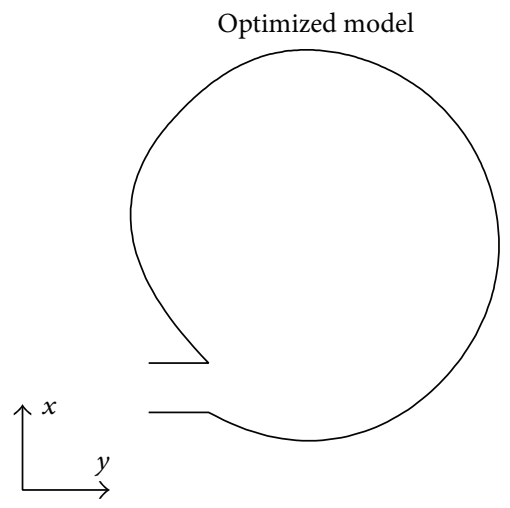

(b)

FIGURE 14: Shape of the best baseline model and the optimized volute geometry.

regarding the volute try to reduce losses. Therefore, an increase in efficiency also increases the total pressure ratio.

Figure 14 shows the geometry of the best baseline model from the initial generation (a) in comparison with the result of the shape optimization (b). Both offer round shapes. However, the optimized model has a sharper angle between the 


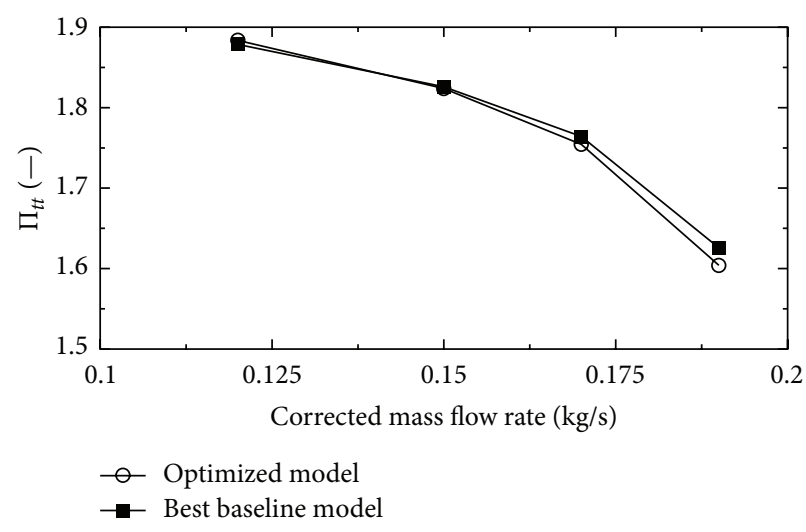

(a)

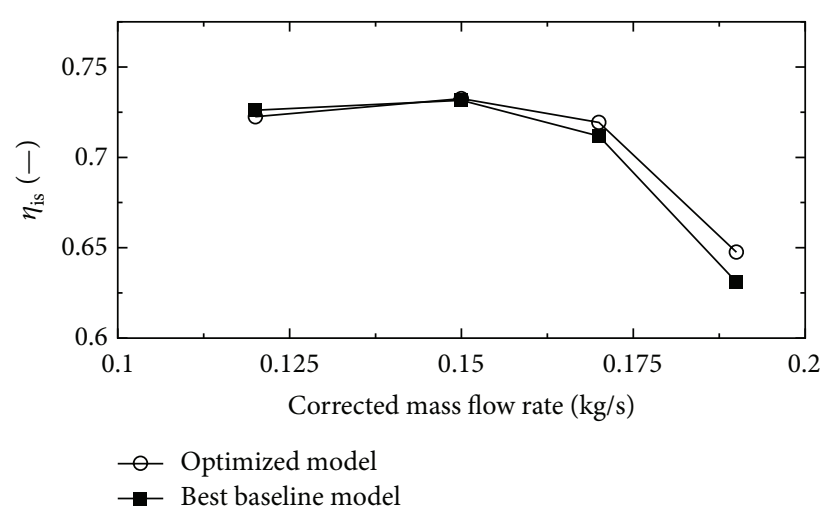

(b)

FIGURE 15: Total pressure ratio (a) and isentropic efficiency (b) at $n=100,000 \mathrm{~min}^{-1}$ for the best baseline and optimized volute shape.

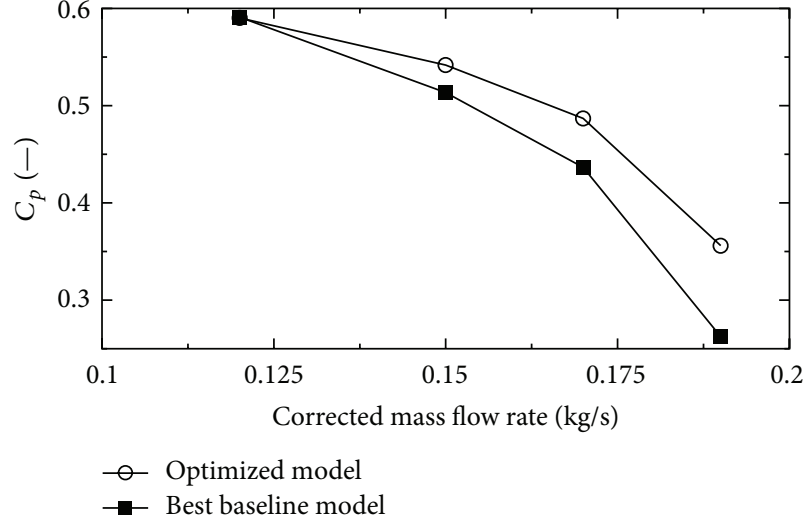

(a)

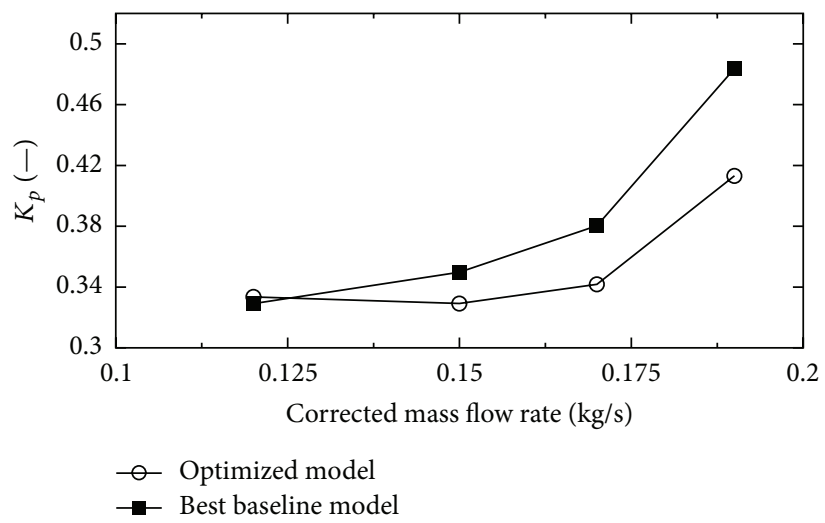

(b)

FIGURE 16: Static pressure recovery coefficient (a) and total pressure loss coefficient (b) at $n=100,000 \mathrm{~min}^{-1}$ for the best baseline and optimized volute shape.

upper diffuser wall and the inner volute wall. Furthermore, the lower wall is curved. These differences result in a slightly lower radial position of the volute channel.

The differences in terms of compressor performance between the shapes close to design point are neglectable (see Figure 15). Low flow velocities result in lower friction losses. Furthermore, the cross-sectional area of the volute is designed for this mass flow rate resulting in a lower influence of the actual shape. In contrast to that the effect at high flow rates is more significant. In particular, the isentropic efficiency and the total pressure ratio of the optimized model are increased by up to $2.5 \%$ at $\dot{m}_{\text {corr }}=0.19 \mathrm{~kg} / \mathrm{s}$.

The static pressure recovery coefficient $C_{p}$ and the total pressure loss coefficient $K_{p}$ solely define the performance of the volute. These quantities are defined between the vaneless diffuser inlet (subscript: in) and the outlet (subscript: out) of the compressor as follows:

$$
\begin{aligned}
C_{p} & =\frac{p_{\text {out }}-p_{\text {in }}}{p_{t, \text { in }}-p_{\text {in }}}, \\
K_{p} & =\frac{p_{t, \text { in }}-p_{t, \text { out }}}{p_{t, \text { in }}-p_{\text {in }}},
\end{aligned}
$$

with the mass-weighted static pressure $p$ and the total pressure $p_{t}$. Comparing the performance of the different volutes close to design point at $\dot{m}_{\text {corr }}=0.12 \mathrm{~kg} / \mathrm{s}$ reveals neglectable differences (see Figure 16). However, at high flow rates, the optimized geometry offers an increase in $C_{p}$ by up to $35 \%$ and lower $K_{p}$ by up to $12 \%$. The best pressure recovery and the lowest total pressure loss can be found at $\dot{m}_{\text {corr }}=$ $(0.12-0.15) \mathrm{kg} / \mathrm{s}$.

The static pressure at different azimuthal angles for the best baseline and the optimized geometry is shown in Figure 17 for an operating point of $0.19 \mathrm{~kg} / \mathrm{s}$. Both models show a similar behaviour. The static pressure at $\phi=1 / 2 \pi$, where the cross-sectional area is the smallest, is higher than downstream the volute channel. Due to the high flow rates, the volute acts like a nozzle. The streamwise development in cross-sectional area of the volute is too small to decelerate the flow. Therefore, the static pressure is decreased along the volute channel. However, the optimized geometry shows an improved pressure recovery compared to the baseline model, in particular at the volute exit $\phi=2 \pi$.

The advantage of the optimized volute geometry is the slightly sharper corner between the upper vaneless diffuser 


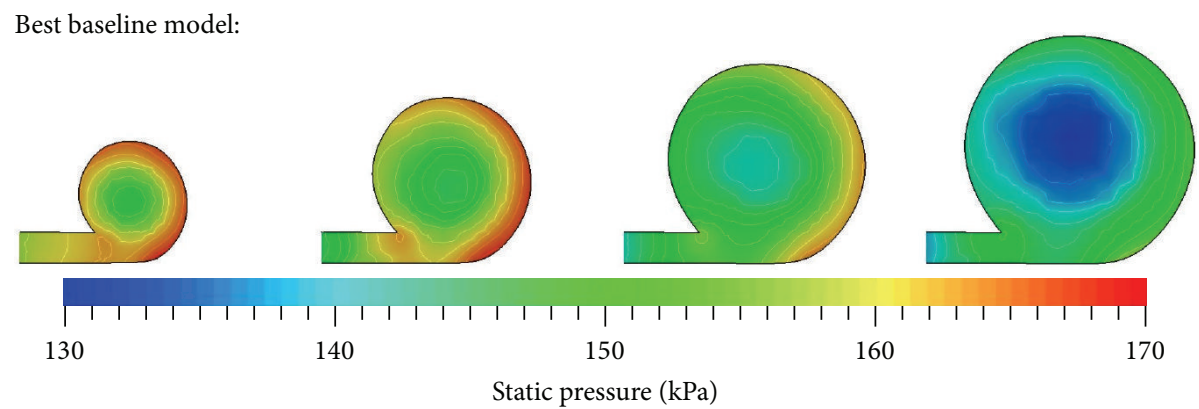

(a)

Optimized model:
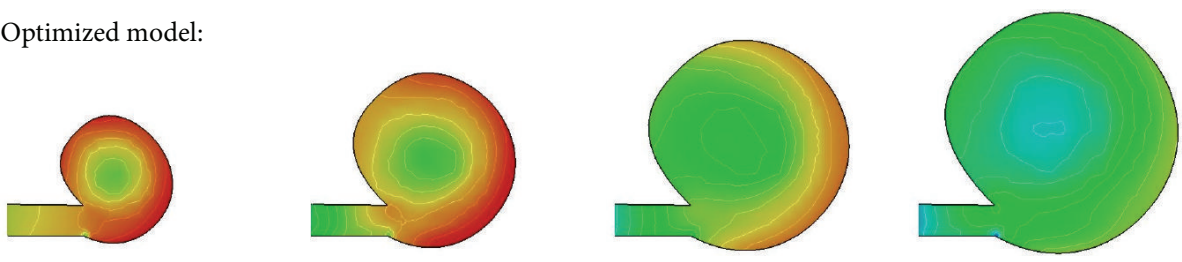

$\theta=1 / 2 \pi$

$\theta=\pi$

$\theta=3 / 2 \pi$

$\theta=2 \pi$

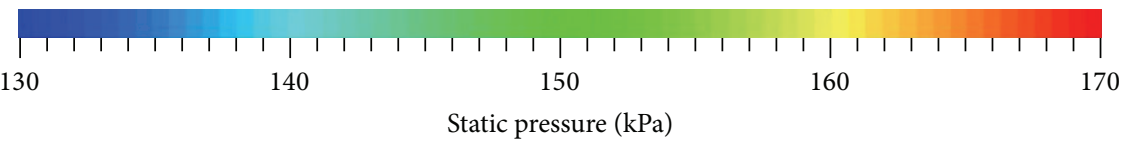

(b)

FIGURE 17: Static pressure at various azimuthal angles $\phi$ for the best baseline model (a) and optimized volute geometry $(\mathrm{b})$ at $\dot{m}_{\text {corr }}=0.19 \mathrm{~kg} / \mathrm{s}$.

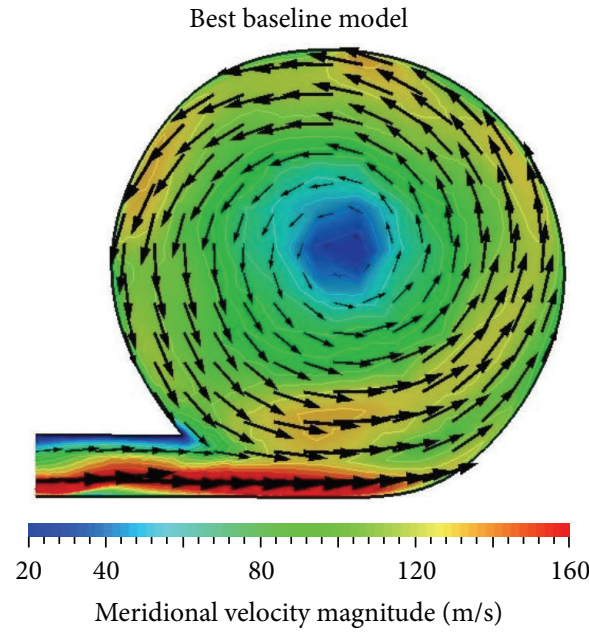

(a)

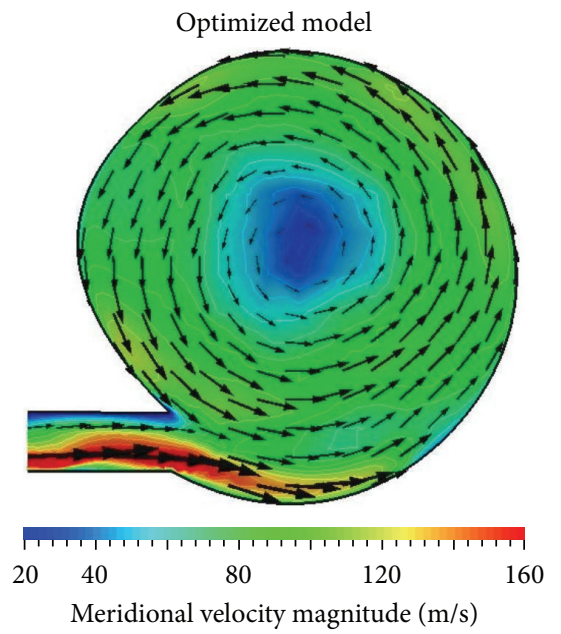

(b)

FIGURE 18: Meridional velocity magnitude at volute exit $\phi=2 \pi$ for the best baseline model (a) and optimized volute geometry $(\mathrm{b})$ at $\dot{m}_{\text {corr }}=$ $0.19 \mathrm{~kg} / \mathrm{s}$.

wall and the inner volute wall (see Figure 18). This supports the vortex rotation within the volute channel and suppresses the development of a secondary vortex in this region. Furthermore, the lower wall is curved which results in a deflection of the jet downstream the volute inlet by the volute channel vortex. Therefore, near-wall velocity gradients are reduced and the average velocity on the volute is reduced. This leads to lower friction losses and slightly reduced turbulence in the volute channel (see Figure 19). The flow velocity of the optimized geometry is slower since the static pressure is higher and therefore also the density. For this reason, a lower flow velocity is sufficient to reach the desired mass flow rate.

\section{Conclusion}

The paper proposed a numerical model for the simulation of the flow inside a centrifugal compressor and performed a 


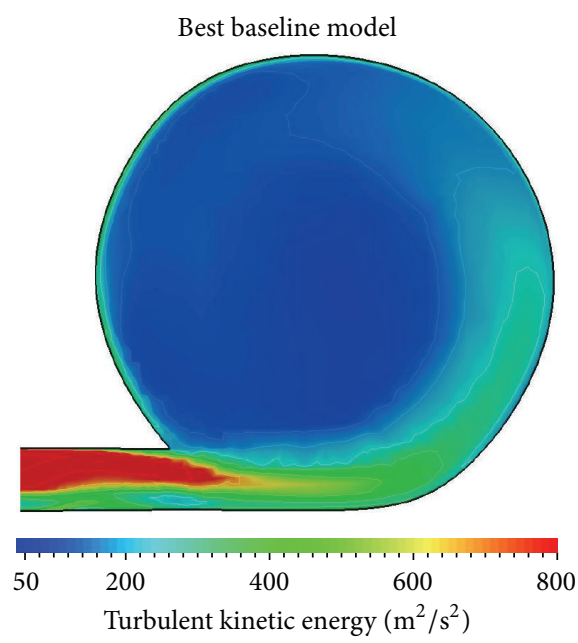

(a)

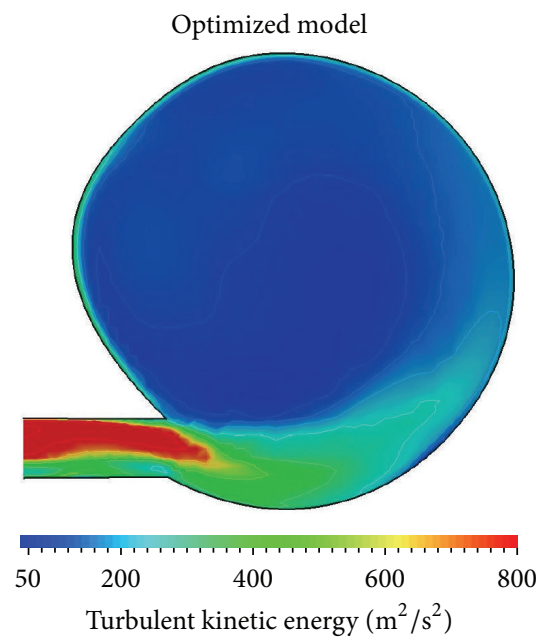

(b)

Figure 19: Turbulent kinetic energy at volute exit $\phi=2 \pi$ for the best baseline model (a) and optimized volute geometry (b) at $\dot{m}_{\text {corr }}$ $=0.19 \mathrm{~kg} / \mathrm{s}$.

genetic optimization to improve the volute and compressor performance. The design parameters consisted of the crosssectional shape of the volute. The surface representation was based on cubic B-splines, where the control points are used as variables. The optimized volute offered a better operating range and an increased total pressure ratio as well as isentropic efficiency. The geometric differences are minor. However, they reveal an important effect onto the volute performance. The static pressure recovery at off-design conditions is raised by up to $32 \%$, while the total pressure loss is reduced over $12 \%$.

\section{Competing Interests}

The authors declare that they have no competing interests.

\section{References}

[1] D. Eckardt, "Detailed flow investigations within a high-speed centrifugal compressor impeller," Journal of Turbomachinery, vol. 98, no. 3, pp. 390-399, 1976.

[2] F. Hellstrom, E. Gutmark, and L. Fuchs, "Large eddy simulation of the unsteady flow in a radial compressor operating near surge," Journal of Turbomachinery, vol. 134, no. 5, Article ID 051006, 10 pages, 2012.

[3] K. U. Ziegler, H. E. Gallus, and R. Niehuis, "A study on impellerdiffuser interaction-part I: influence on the performance," Journal of Turbomachinery, vol. 125, pp. 173-182, 2003.

[4] K. U. Ziegler, H. E. Gallus, and R. Niehuis, "A study on impellerdiffuser interaction-part II: detailed flow analysis," Journal of Turbomachinery, vol. 125, no. 1, pp. 183-192, 2003.

[5] E. Ayder and R. Van den Braembussche, "Experimental study of the swirling flow in the internal volute of a centrifugal compressor," in Proceedings of the International Gas Turbine of Aeroengine Congress \& Exhibition, pp. 1-8, Orlando, Fla, USA, June 1991.

[6] R. C. Worster, "The flow in volutes and its effect on centrifugal pump performance," Proceedings of the Institution of Mechanical Engineers, vol. 177, no. 1, pp. 843-875, 1963.

[7] C. Abdelmadjid, S.-A. Mohamed, and B. Boussad, "CFD analysis of the volute geometry effect on the turbulent air flow through the turbocharger compressor," Energy Procedia, vol. 36, pp. 746-755, 2013.

[8] H. Chen, D. Tong, and X. Wang, "Vortices and performance of internal and external volutes," in Proceedings of ASME Turbo Expo 2015: Turbine Technical Conference and Exposition, pp. 1-8, Montreal, Canada, June 2015.

[9] J. H. Kim, K. T. Oh, K. B. Pyun, C. K. Kim, Y. S. Choi, and J. Y. Yoon, "Design optimization of a centrifugal pump impeller and volute using computational fluid dynamics," IOP Conference Series: Earth and Environmental Science, vol. 15, part 3, Article ID 032025, 2012

[10] M. Mojaddam, A. H. Benisi, and M. R. Movahhedy, "Investigation on effect of centrifugal compressor volute cross-section shape on performance and flow field," in Proceedings of the ASME Turbo Expo 2012: Turbine Technical Conference and Exposition, pp. 1-10, Copenhagen, Denmark, June 2012.

[11] S. Yang, F. Kong, and B. Chen, "Research on pump volute design method using CFD," International Journal of Rotating Machinery, vol. 2011, Article ID 137860, 7 pages, 2011.

[12] Elsevier, “Scopus Journal Index,” 2015, http://www.scopus.com.

[13] C.-M. Jang, J.-S. Lee, and S.-H. Yang, "Performance evaluation of a centrifugal blower using optimal design method," in Proceedings of the ASME Fluids Engineering Division Summer Meeting (FEDSM '13), pp. 1-7, July 2013.

[14] B. D. Baloni, Y. Pathak, and S. A. Channiwala, "Centrifugal blower volute optimization based on Taguchi method," Computers \& Fluids, vol. 112, pp. 72-78, 2015.

[15] J. Huang, S. Xu, H. Liu, and X. Wang, "Robust performance optimization of centrifugal compressor volute with a rectangular cross-section," in Proceedings of the ASME Turbo Expo 2015: Power for Land, Sea and Air, pp. 1-11, Montreal, Canada, June 2015.

[16] OpenFOAM Foundation Ldt, OpenFOAM Version 2.4.0, 2015, http://www.openfoam.org.

[17] M. Heinrich and R. Schwarze, "All-mach number density based flow solver for OpenFOAM," in Proceedings of the ASME Turbo 
Expo 2014: Turbine Technical Conference and Exposition, pp. 110, Düsseldorf, Germany, June 2014.

[18] K. Kitamura and E. Shima, "Improvements of simple lowdissipation AUSM against shock instabilities in consideration of interfacial speed of sound," in Proceedings of the European Conference on Computational Fluid Dynamics, Lisbon, Portugal, June 2010.

[19] E. Shima, "A compressible CFD method for flow with sound from very low mach number to supersonic," in Proceedings of the 6th International Colloquium on: Bluff Bodies Aersodynamics \& Applications (BBAA VI '08), pp. 20-24, Milano, Italy, July 2008.

[20] P. E. Smirnov and F. R. Menter, "Sensitization of the SST turbulence model to rotation and curvature by applying the Spalart-Shur correction term," Journal of Turbomachinery, vol. 131, no. 4, pp. 1-8, 2009.

[21] B. M. Adams, M. S. Ebeida, M. S. Eldred et al., "Dakota, a multilevel parallel object-oriented framework for design optimization, parameter estimation, uncertainty quantification, and sensitivity analysis: version 6.0 user's manual," Tech. Rep., Sandia National Laboratories, Albuquerque, NM, USA, 2014.

[22] Z. Sun, C. Tan, and D. Zhang, "Flow field structures of the impeller backside cavity and its influences on the centrifugal compressor," in Proceedings of the ASME Turbine Technical Conference and Exposition, vol. 7, pp. 1349-1360, Orlando, Fla, USA, June 2009.

[23] D. Bohn, T. Heuer, and K. Kusterer, "Conjugate flow and heat transfer investigation of a turbo charger," Journal of Engineering for Gas Turbines and Power, vol. 127, no. 3, pp. 663-669, 2005.

[24] T. Verstraete, Z. Alsalihi, and R. A. Van den Braembussche, "Numerical study of the heat transfer in micro gas turbines," Journal of Turbomachinery, vol. 129, no. 4, pp. 835-841, 2007.

[25] O. Baris and F. Mendonca, "Automotive turbocharger compressor CFD and extension towards incorporating installation effects," in Proceedings of the ASME Turbo Expo 2011: Turbine Technical Conference and Exposition, 2011.

[26] B. D. Baloni and S. A. Channiwala, "Design and analysis of volute casing: a review," in Proceedings of the ASME Turbo Expo 2012: Turbine Technical Conference and Exposition, pp. 657-664, Copenhagen, Denmark, June 2012. 


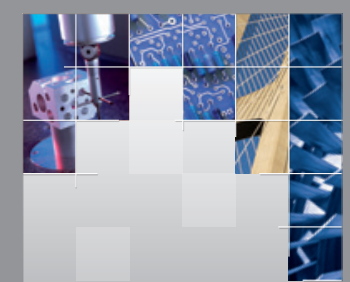

\section{Enfincering}
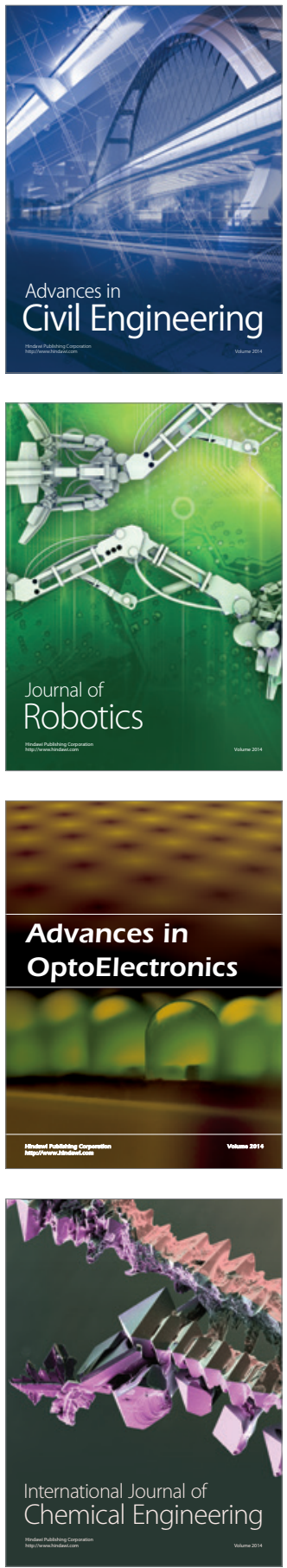

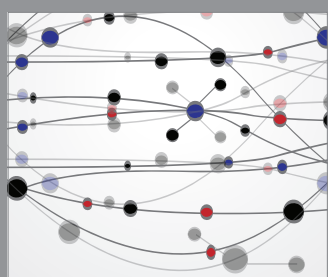

The Scientific World Journal

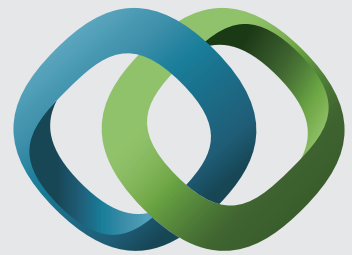

\section{Hindawi}

Submit your manuscripts at

http://www.hindawi.com
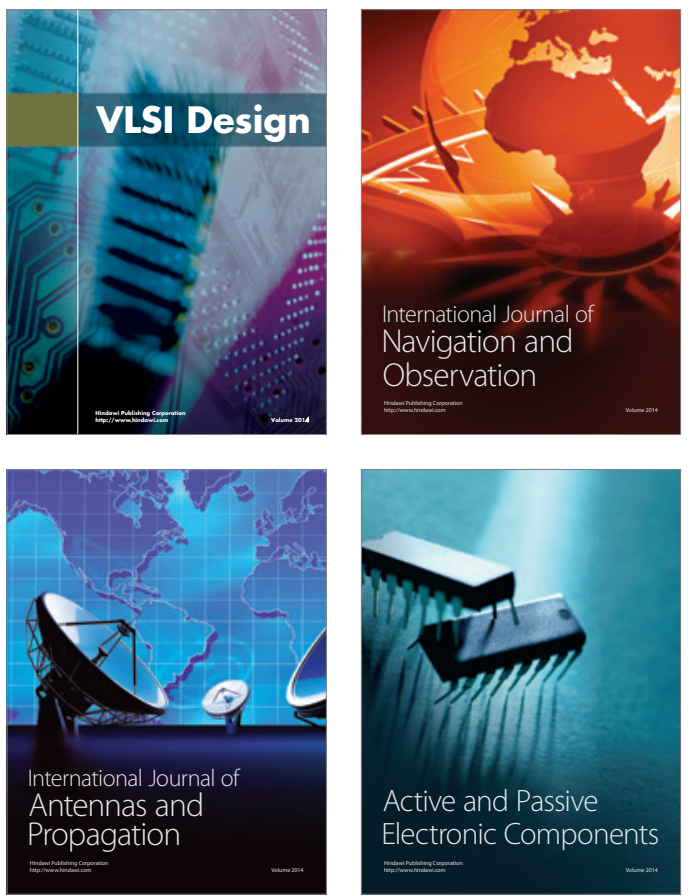
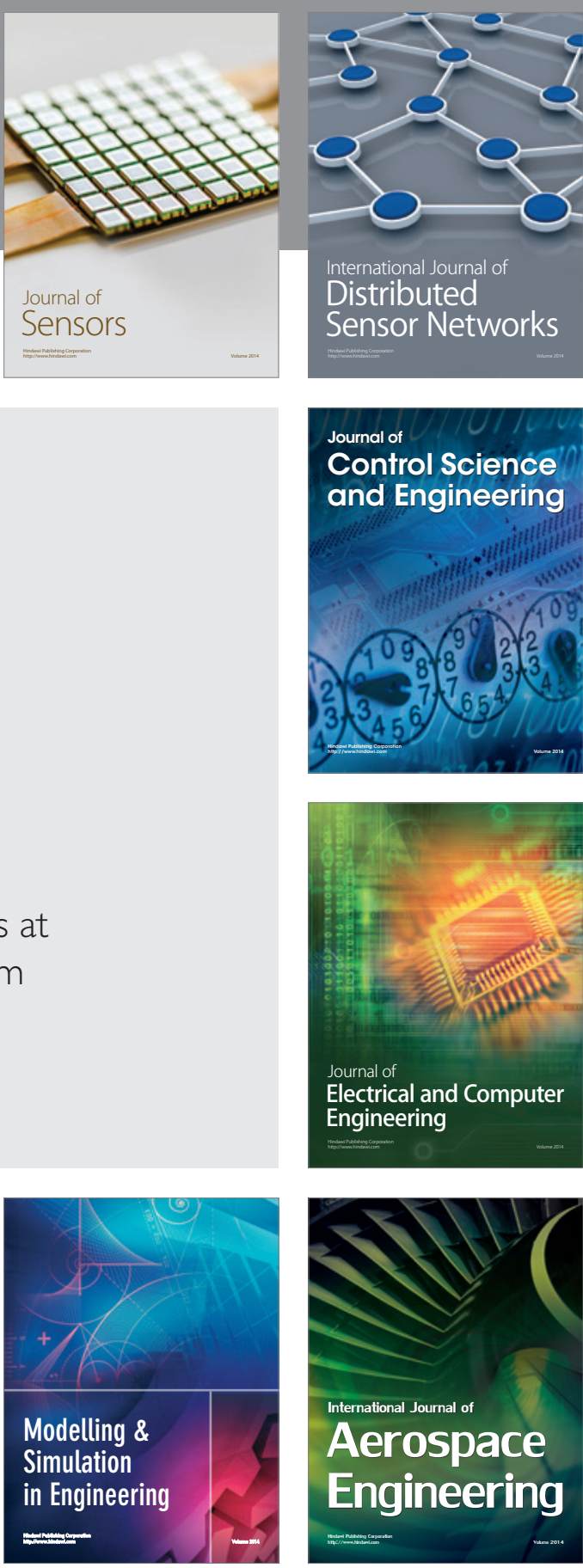

International Journal of

Distributed

Sensor Networks

Journal of

Control Science

and Engineering
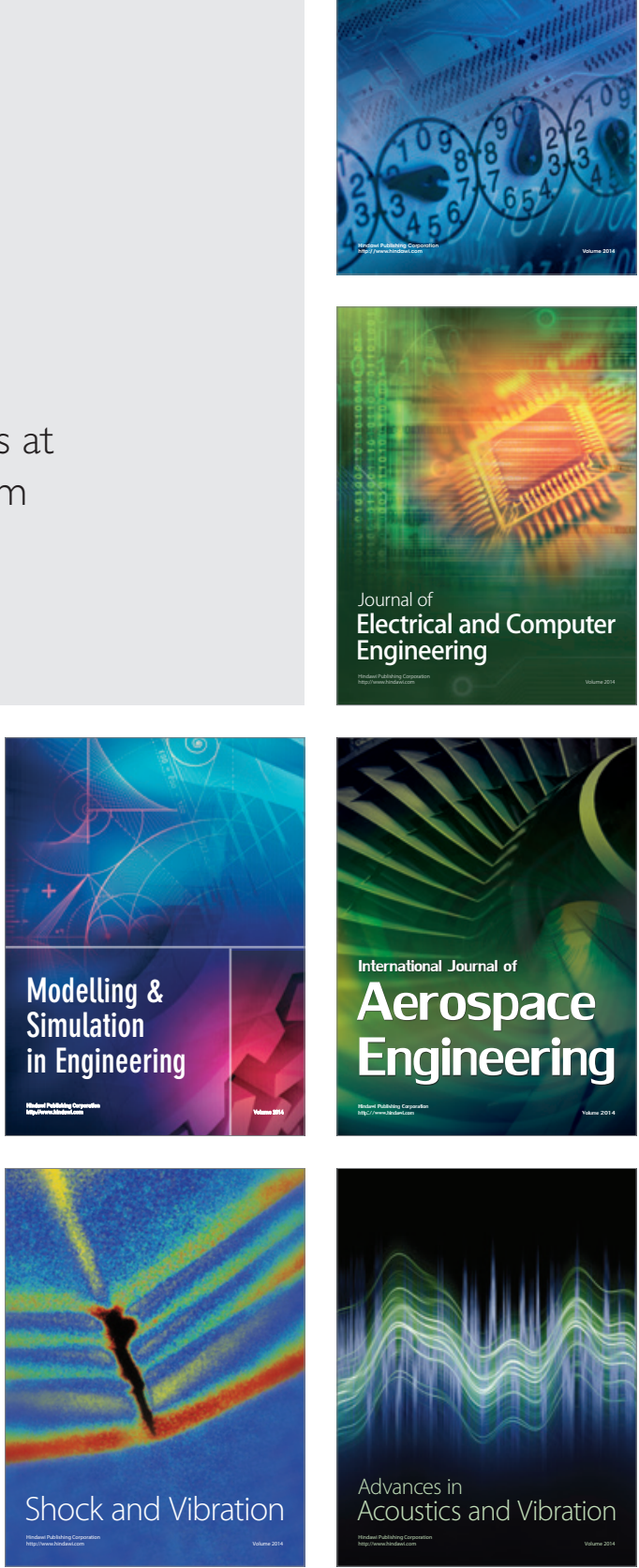